\title{
Shape and operation optimization of a thick-walled power boiler component
}

\author{
Piotr Duda ${ }^{1, *}$, and Mirosław W. Mrzygłód ${ }^{2}$ \\ ${ }^{1}$ Institute of Thermal and Process Engineering, Cracow University of Technology, Al. Jana Pawła II 37, 31-864 Kraków, Poland \\ ${ }^{2}$ Department of Mechanics and Machine Design, Opole University of Technology, Mikolajczyka 5, 45-271 Opole, Poland
}

\begin{abstract}
The aim of this article is the proposition of a two-stage optimization of power boiler components subjected to transient thermo-mechanical loads. As the basis of transient load regime, the TRD 301 regulations are used. In the first stage of an investigation, the genetic algorithms are used to determine the optimal shape of the structure. As optimization constraints, the maximum total stresses in the whole cycle are assumed. In the next stage, the method for the start-up and shut-down operation optimization is shown. The problem is defined to minimize the time of start-up and shut-down processes with allowable stress constraints according to TRD regulations. These new methods are of high practical significance and can be implemented throughout the industry, wherever heating and cooling processes take place. The obtained optimization results translate directly into the power unit energy effectiveness because the shut-down and start-up losses are reduced.
\end{abstract}

\section{Introduction}

A challenge of the XXI century is providing abundant and affordable electricity to meet the need of a growing global population, while lowering and controlling $\mathrm{CO} 2$ emissions. Both of these goals can be met through the development of highly efficient technologies of power units. During exploitation of power boiler components, high thermal stresses occur. The cyclic character of such stresses causes low-cyclic fatigue, which may lead to fracture. Manufacturers of such equipment advise keeping within prescribed limits for maximum heating and cooling rates. A long start-up and shut-down operation time of power boilers causes big losses in the electricity generation. On the other hand, rapid transient operations of power boilers cause high temperature differences and thermal stresses in the construction elements [1]. Methods to find optimum operating parameters, that can assure safe heating and cooling processes can be found in $[2,3,4]$. Reducing the thickness of the walls of boiler components while maintaining the level of stress during the cycle within established limits can bring additionally significant economic benefits. Furthermore, it can improve the thermomechanical characteristics of the device.

The aim of this article will be the proposition of a method for lightweight optimization of power boiler components subjected to transient thermo-mechanical loads according to TRD 301 regulations [5]. As optimization constraints, the maximum total stresses are assumed. Next, the method for the start-up and shutdown operation optimization is shown. The problem is defined to minimize the time of start-up and shut-down processes with stress constraints.

Examples of cyclic load thermo-mechanical structural optimization are rarely published. Analytical expressions for shape design sensitivities in the optimization of engine exhaust manifold with respect to its geometry were presented in the work of Yang and Poe [6]. The manifold was formulated as a linear heat transfer and thermo-elasticity problem in a variational form. An example of design and optimization of high-temperature engine components was presented in the paper of Tappeta et al. [7]. These authors used multiple analysis codes and response surface methodology for the design and optimization of large-scale real-life engineering systems.

The majority of available examples of the application of modern structural optimization of power block devices refer to the steady state. Wen et al. [8] present an example of structural optimization of cellular cores of metallic sandwiches being subjected to laminar forced convection at fixed pumping power. Yoon et al. [9] use isogeometric analysis to solve the heat conduction equation and compute shape design sensitivity coefficients. Xie et al. [10] employ a globally convergent method of moving asymptotes (GCMMA) in the lightweight optimization of a corrugated sandwich panel using heat transfer and thermo-mechanical analyses. Xiao and Huang [11] combine particle swarm optimization (PSO) with the finite element method (FEM) to find the optimal design for achieving rapid and uniform mold heating system in RTCM molds. Nowak and Rusin [12] present the problem of shape optimisation of selected areas of the rotor of the high

\footnotetext{
* Corresponding author: pduda@mech.pk.edu.pl
} 
pressure part of an ultrasupercritical steam turbine together with the optimisation of the turbine startup method.

In this article, the genetic algorithms will be employed to determine the optimal dimensions of a thick-walled boiler component. Next, the optimization of the start-up and shut-down operations is proposed by the use of the successive quadratic programming algorithm [13].

\section{Problem formulation}

The heat transfer in a thick-walled boiler component is controlled by heat conduction. The transient heat conduction equation in three-dimensional Cartesian coordinates reads:

$$
\frac{\partial}{\partial x}\left(k \frac{\partial T}{\partial x}\right)+\frac{\partial}{\partial y}\left(k \frac{\partial T}{\partial y}\right)+\frac{\partial}{\partial z}\left(k \frac{\partial T}{\partial z}\right)=\rho c \frac{\partial T}{\partial t}
$$

where $k$ is the thermal conductivity, $T$ is the temperature, $\rho$ is the density, $c$ is the specific heat capacity, and $t$ is the time instant.

The highest stresses in the component should be limited to allowable values. This restriction can be captured by a simple structural analysis based on elementary equations of thermoelasticity.

$$
\begin{gathered}
\sigma_{i j, j}+f_{i}=0 \\
\varepsilon_{i j}=\frac{1}{2}\left(u_{i, j}+u_{j, i}\right) \\
\sigma_{i j}=2 G \varepsilon_{i j}+\lambda \varepsilon_{k k} \delta_{i j}+\beta T \delta_{i j}
\end{gathered}
$$

The equation (2) is the force equilibrium equation, (3) is the geometric equation, and (4) is the constitutive equation. The variable $\sigma$ is the stress tensor, $\mathbf{f}$ is the body forces vector, $\mathbf{u}$ is the displacement vector, $\boldsymbol{\varepsilon}$ is the strain tensor, $G$ and $\lambda$ are the Lamé constants, $\beta$ is the thermal expansion coefficient and $\delta_{i j}$ is the Kronecker delta.

Set of equations (1-4) should be satisfied throughout the boiler component volume, which can be designed as $V$. Transient heat conduction problems are initialboundary problems and appropriate initial and boundary conditions should be defined. Initial conditions, are temperature values of a component at its initial moment. There are four basic types of boundary conditions, but usually two of them are used. One part of the component is insulated and 2nd order boundary conditions simplifies to the form

$$
\left.\frac{\partial T}{\partial n}\right|_{A}=0
$$

where $n$ is the external normal direction to the component edge surface $A$. The second part of boundary is in contact with fluid and the boundary condition of 3rd order can be written

$$
-\left.k \frac{\partial T}{\partial n}\right|_{A}=h\left[\left.T(x, y, z, t)\right|_{A}-T_{f}\right]
$$

where $h$ is heat transfer coefficient and $T_{f}$ is fluid temperature. In general both $h$ and $T_{f}$ can vary in time and space. Equations of thermoelasticity (2-4) should be accompanied by boundary conditions defined by displacement or force. Finite element method will be used for discretization of the above equations.

\subsection{Shape optimization}

The shape of a thick-walled boiler component determine the level of stress that arise at different phases of its operation. Reducing the thickness of the component will result in an increase in stresses caused by internal pressure but will reduce thermal stresses. So the thickness of the walls can be reduced without increasing total stresses. The advantage of the component with thin walls is its lower weight, volume, and it can be faster heated or cooled.

In the optimization process, the design space search is performed using evolutionary algorithms and a parametric FE model (see Fig. 1). As the tool of the investigation, the Evolutionary Optimization System (EOS) software has been chosen $[14,15]$. The EOS is designed to solve single and multicriteria optimization problems for nonlinear programming problems. The single criterion optimization thermo-mechanical problems can be formulated as follows:

- find the vector of decision variables (e.g. dimensions of a thick-walled boiler component)

$$
\mathbf{y}_{\mathrm{k}}=\left[y_{1}, y_{2}, \ldots, y_{K}\right]
$$

which will satisfy the $J=2$ inequality constraints:

- for the heating operation

$$
g_{1}(\mathbf{x})=S_{3 \min }(\mathbf{x})-\bar{\sigma}_{a} \geq 0
$$

- for the cooling operation

$$
g_{2}(\mathbf{x})=\widehat{\sigma}_{a}-S_{1 \max }(\mathbf{x}) \geq 0
$$

where $g_{j}(\mathbf{x}), j=1,2$ are the functions of constrains; $S_{3 \text { min }}$ denotes the lowest third principal stress in component during the heating process, $S_{1 \max }$ is the highest first principal stress in component during the cooling process, $\bar{\sigma}_{a}$ and $\bar{\sigma}_{a}$ denote allowable stress for the heating and cooling operations. Both values $S_{3 \min }$ and $\bar{\sigma}_{a}$ are compressive stresses and are negative. Tensile stresses $S_{1 \max }$ and $\hat{\sigma}_{a}$ are positive.

For the objective function, the volume of the structure has been chosen. The algorithm can be adjusted to the investigated object using the following control parameters [14]: population size $P J$, parameter for the operation of crossing $p_{c}$, parameter for the operation of mutation $p_{m}$, number of generations $L_{g}$. 


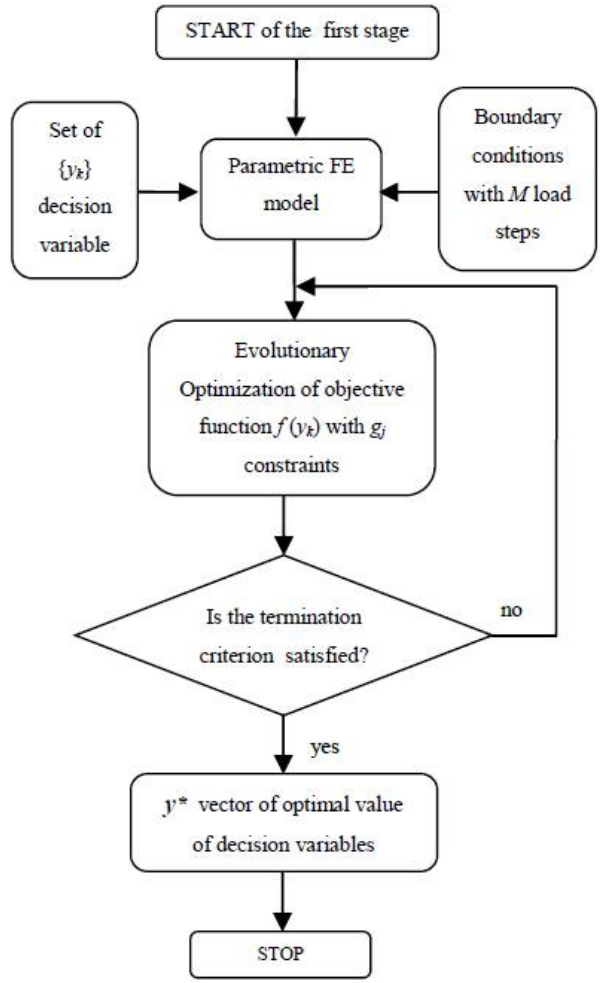

Fig. 1. Proposed parametric GA optimization methodology.

\subsection{Optimization of start-up and shut-down operations}

The aim of this optimization is to find the fluid transient temperature $T_{f}(t)$, which heats or cools a thick-walled boiler component in such a way that the total time of heating and cooling operation is the lowest and maximum tensile and compressive total stresses in component do not exceed the allowable values during entire process.

Set of equations (1-4) should be satisfied throughout the boiler component volume, which can be designed as $V$. Transient heat conduction problems are initialboundary problems and appropriate initial and boundary conditions should be defined. Initial conditions, are temperature values of a component at its initial moment. There are four basic types of boundary conditions, but usually two of them are used. One part of the component is insulated and 2nd order boundary conditions simplifies to the form

The fluid temperature $T_{f}(t)$ is assumed as a piecewise linear function. It consists of initial fluid temperature $T_{f_{1}}$, temperature change rate $v_{T_{1}}$, second fluid temperature $T_{f_{2}}$ and final temperature change rate $v_{T_{2}}$. The second fluid temperature change occurs immediately after obtaining the superheated steam. During the heating process, fluid enters the component of initial temperature $T_{0}$. For the cooling process, fluid enters a thick-walled boiler component of working temperature $T_{w}$. The proposed course of the fluid temperature is presented in Fig. 2.
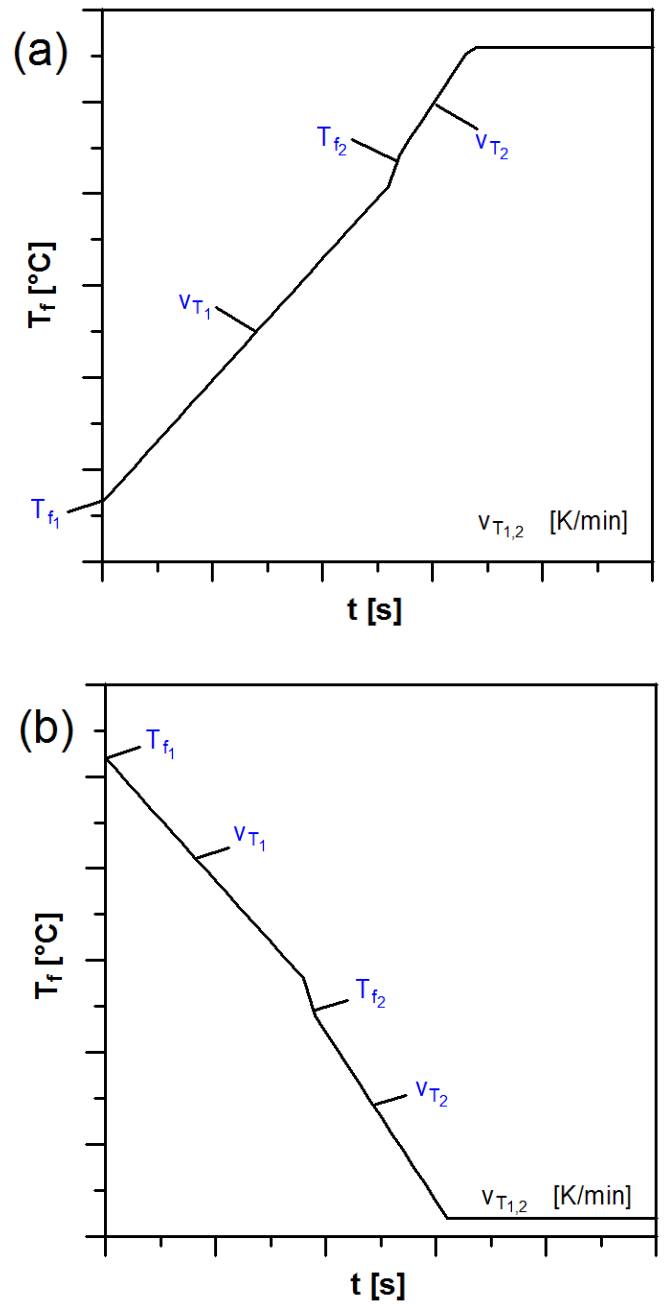

Fig. 2. The chosen function of fluid temperature during heating (a) and cooling operation (b).

A nonlinear optimization problem with inequality constraints is formulated. The aim is to find the best vector $\mathbf{x}=\left\{T_{f_{1}}, v_{T_{1}}, T_{f_{2}}, v_{T_{2}}\right\}$. The optimum vector $\mathbf{x}$ should minimize the objective function $S$, which is defined as a total time of heating or cooling operation $t_{t o t}$

$$
S(\mathbf{x})=t_{t o t}
$$

while limiting inequality constraints (8-9). The design vector $\mathbf{x}$ changes in the range

$$
\mathbf{x}_{l} \leq \mathbf{x} \leq \mathbf{x}_{u}
$$

where subscripts $l$ and $u$ denote lower and upper bounds of searched parameters, respectively. A general nonlinear programming problem is solved using the successive quadratic programming algorithm [13].

The total stress can be calculated adding the influence of inner pressure. When fluid is the pressurized water or superheated steam, the pressure value $p_{f}$ is independent of temperature. In the case of saturated water, steam-water mixture or saturated steam, $p_{f}$ is the saturation pressure $p_{s}$ defined as the function of fluid temperature $T_{f}(t)$ in ${ }^{\circ} \mathrm{C}$. 


\section{Numerical example}

Shape and operation optimization will be presented for a T-pipe, which is the part of the fresh and preheated steam pipeline in the $360 \mathrm{MW}$ power plant. It was designed for the pressure $p_{w}=18 \mathrm{MPa}$ and steam temperature $T_{w}=540^{\circ} \mathrm{C}$. It weighs $1378 \mathrm{~kg}$. The geometry of the T-pipe is presented in Fig. 3. The T-pipe is made of alloy steel 14 MoV63 (13 HMF) [16].

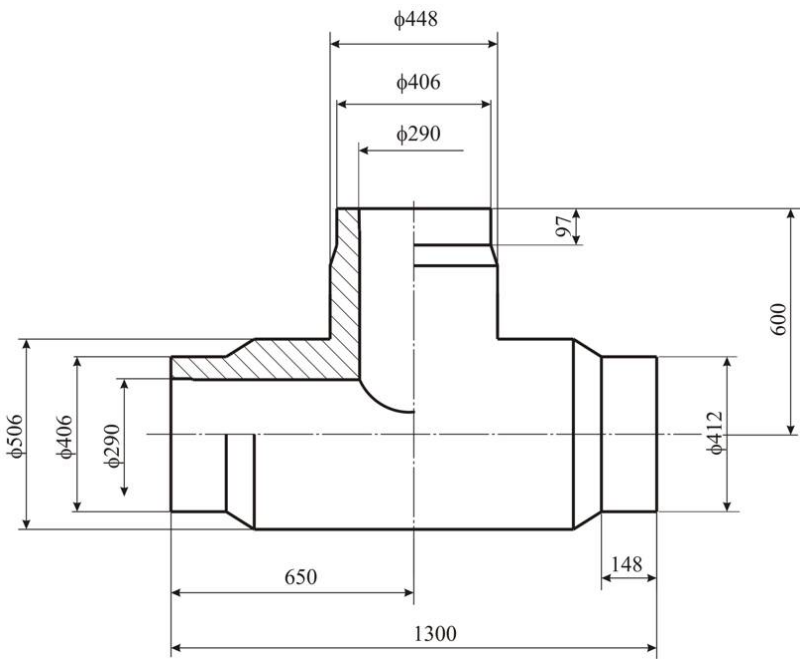

Fig. 3. Geometry of the T-pipe.

TRD301 code [5] can regulate the method of heating or cooling the chosen thick-walled boiler component. It assumes a quasi-steady state and one-dimensional temperature distribution through the wall of construction element. For the chosen T-pipe, the following stress concentration coefficients are considered: $\alpha_{p}=3.2$, $\alpha_{T}=2$. The calculated allowable stresses for heating and cooling operations according to TRD301 are $\breve{\sigma}_{a}=-214.4 \mathrm{MPa}, \widehat{\sigma}_{a}=303 \mathrm{MPa}$. The allowable rates of temperature change at the beginning and end of the process are obtained based on allowable stresses: $v_{T 1(T R D)}=2.119 \mathrm{~K} / \mathrm{min}, v_{T 2(T R D)}=2.993 \mathrm{~K} / \mathrm{min}$ - for heating and $\quad v_{T 1(T R D)}=-2.119 \mathrm{~K} / \mathrm{min}, v_{T 2(T R D)}=$ $=-2.993 \mathrm{~K} / \mathrm{min}-$ for cooling.

Once the pressure at the beginning of the heating process equals $0 \mathrm{MPa}$ and the pressure at the end of the heating process equals $p_{w}$, the fluid temperature can be calculated from the equation:

$$
\frac{d T_{f}}{d t}=v_{T 1(T R D)}+\frac{v_{T 2(T R D)}-v_{T 1(T R D)}}{p_{w}} p_{f}\left(T_{f}\right)
$$

In a similar way, the fluid temperature for the cooling process can be determined. The calculated fluid temperature and pressure for heating and cooling operation are presented in Fig. 4.

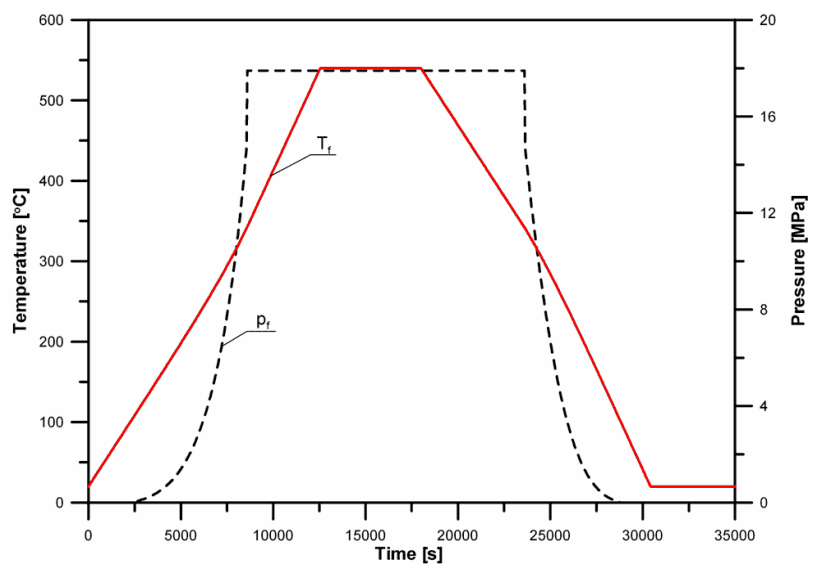

Fig. 4. The calculated medium temperature and medium pressure according to TRD 301.

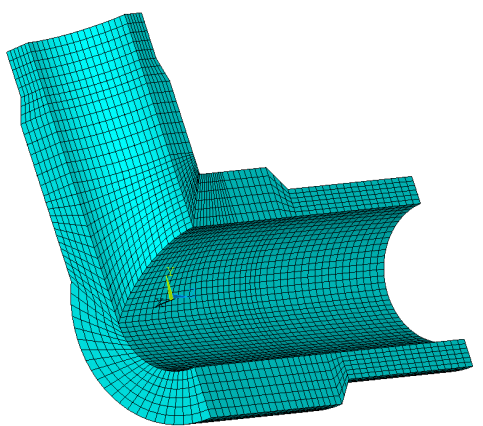

Fig. 5. T-pipe division into finite elements.

One quarter of the T-pipe was divided into 20-node finite brick elements based on the symmetry conditions. This is shown in Fig. 5. Temperature and stress distribution in the T pipe is calculated using ANSYS software [17]. Temperature distribution in time and space is calculated assuming that the heat transfer coefficient on the inner surfaces of the T-pipe equals $1000 \mathrm{~W} /\left(\mathrm{m}^{2} \mathrm{~K}\right)$ [18]. Next, stress analyses are carried out. The stress model is constrained to ensure symmetry conditions. It allows for the possibility of unrestricted lengthening in the direction of the horizontal and vertical pipe. Additionally, the surfaces connecting the T-pipe with the pipeline must be planes and the calculated temperature distribution in space and time is used as the body load in the stress model.

The maximum value of equivalent stress and the first principal stress caused by pressure and temperature occurs at about $23100 \mathrm{~s}$ and equals $\sigma_{H M H \max }=246.8 \mathrm{MPa}$ and $\mathrm{S}_{1 \max }=229.2 \mathrm{MPa}$ respectively. The maximum compressive stress can be estimated by the minimum value of the third principal stress. The minimum value of the third principal stress occurs at about $10300 \mathrm{~s}$ and equals $\mathrm{S}_{3 \min }=-139.1 \mathrm{MPa}$. Throughout the entire cycle the allowable stresses $\widetilde{\sigma}_{a}=-214.4 \mathrm{MPa}, \bar{\sigma}_{a}=303 \mathrm{MPa}$ are not exceeded. Principal stresses $\mathrm{S}_{1 \text { max }}$ and $\mathrm{S}_{3 \min }$ will be adopted for the bounds of constraints during the Tpipe shape optimization.

\subsection{Shape optimization}

The optimization problem is defined to minimize the volume (mass) of the structure assuming for the 
following allowable stresses: $\quad \breve{\sigma}_{a}=-139.1 \mathrm{MPa}$, $\widehat{\sigma}_{a}=229.2 \mathrm{MPa}$.

In the optimization framework, the solution is searched for in 4-dimensional design variable space $(2$ radii and 2 lengths of the T-pipe reinforcements - see Fig. 6).

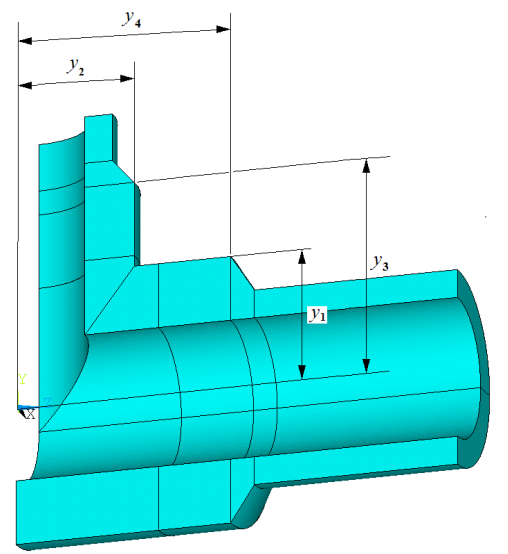

Fig. 6. The 4-dimensional design variable space of GA shape optimization.

For decision variables, the following range of variation is proposed: $y_{1}=\{210 \mathrm{~mm}, 253 \mathrm{~mm}\}, y_{2}=$ $\{205 \mathrm{~mm}, 224 \mathrm{~mm}\}, y_{3}=\{380 \mathrm{~mm}, 680 \mathrm{~mm}\}, y_{4}=\{360$ $\mathrm{mm}, 590 \mathrm{~mm}$.

The parametric FE model is subjected to GA optimization by Evolutionary Optimization System (EOS) software [14]. Moreover, the following steering parameters of EOS are chosen: population size $P J=50$, number of generations $N=25$, probability of crossover $p_{c}=0.7$, probability of mutation $p_{m}=0.06$. The EOS software is connected with the FE program ANSYS for performing batch processing calculations of the objective function value and stress constraints. The best solution $\mathbf{y}^{*}\left(y_{1} *=237.59 \mathrm{~mm}, y_{2}{ }^{*}=215.75 \mathrm{~mm}, y_{3}^{*}=548.81\right.$ $\mathrm{mm}, y_{4}^{*}=366.84 \mathrm{~mm}$ ) is about $10 \%$ lighter than the original one.

Fig. 7 presents $S_{1 \max }$ - maximum values of the first principal stresses in the whole T-pipe over the load time history for the original and optimal shape. Fig. 8 shows $S_{3 \text { min }}$ - minimum values of the third principal stresses in the T-pipe for the both geometries. Modifying the shape does not result in an increase in the maximum stress values in time and space. The highest recorded first principal stress for the optimal shape equals $226.9 \mathrm{MPa}$ and is lower than maximum value for the original shape, which equals $229.2 \mathrm{MPa}$. Additionally, the value of $S_{1 \max }$ for modified geometry is lower than for the original one during almost the whole heating and cooling process (0 s - $13300 \mathrm{~s}$ and $22800 \mathrm{~s}-35000 \mathrm{~s})$. The maximum compressive stresses in the modified geometry are smaller than in the original T-pipe during the whole cycle. Lower stress values recorded during transient processes make it possible to speed up these processes and reduce the shut-down and start-up losses.

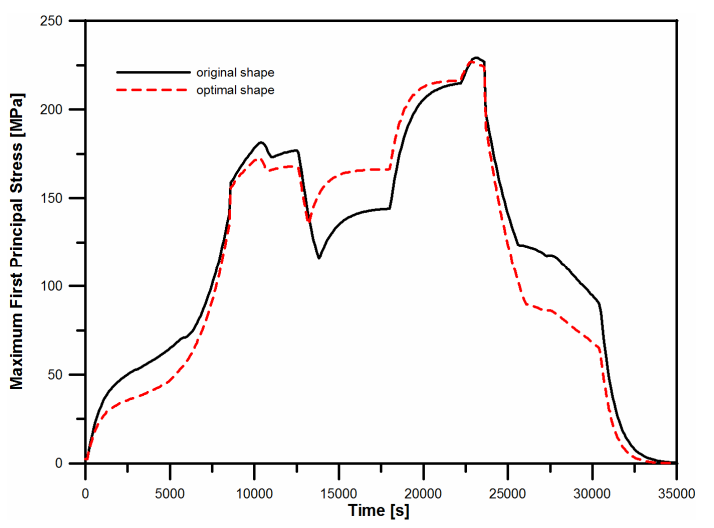

Fig. 7. Maximum first principal stress in the whole structure versus time.

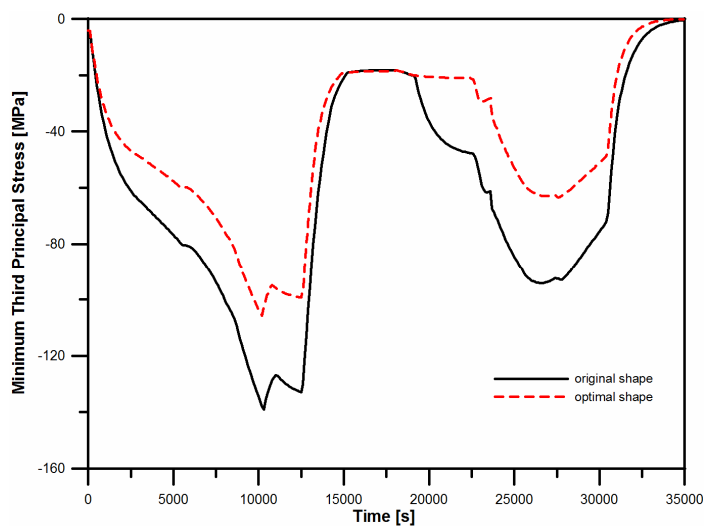

Fig. 8. Minimum third principal stress in the whole structure versus time.

\subsection{Optimization of start-up and shut-down operations}

The heating process optimization is carried out for the Tpipe with the modified shape, which was obtained in the previous Section. The optimization problem is to minimize the start-up process time with the constraint $\breve{\sigma}_{a}=-214.4 \mathrm{MPa}$. Vector of searched parameters $\mathbf{x}$ consists of $\left\{T_{f_{1}}, v_{T_{1}}, T_{f_{2}}, v_{T_{2}}\right\}$. For the lower and upper bounds of the searched-for parameters, in vector $\mathbf{x}$, the values of $\left\{0^{\circ} \mathrm{C}, 0 \mathrm{~K} / \mathrm{min}, 0^{\circ} \mathrm{C}, 0 \mathrm{~K} / \mathrm{min}\right\}$ and $\left\{250^{\circ} \mathrm{C}\right.$, $\left.40 \mathrm{~K} / \mathrm{min}, 540^{\circ} \mathrm{C}, 40 \mathrm{~K} / \mathrm{min}\right\}$ are selected. The starting vector is assumed as $\left\{100^{\circ} \mathrm{C}, 4 \mathrm{~K} / \mathrm{min}, 300^{\circ} \mathrm{C}, 4 \mathrm{~K} / \mathrm{min}\right\}$. Using the proposed optimization method, which is presented in Section 2.2 , the following parameters are found $\left\{147.1^{\circ} \mathrm{C}\right.$, $\left.7.01 \mathrm{~K} / \mathrm{min}, 359.97^{\circ} \mathrm{C}, 6.27 \mathrm{~K} / \mathrm{min}\right\}$. Start-up time is reduced from $12600 \mathrm{~s}$ to $3622 \mathrm{~s}$ as can be seen in Fig.9. Fig. 10 presents $S_{1 \max }$ - maximum values of the first principal stresses and $S_{3 \min }$ - minimum values of the third principal stresses in the whole T-pipe over the start-up load history. The start-up operation based on the proposed optimization method causes higher stresses than the heating according to TRD301. However, all stresses are limited to the allowable values. 


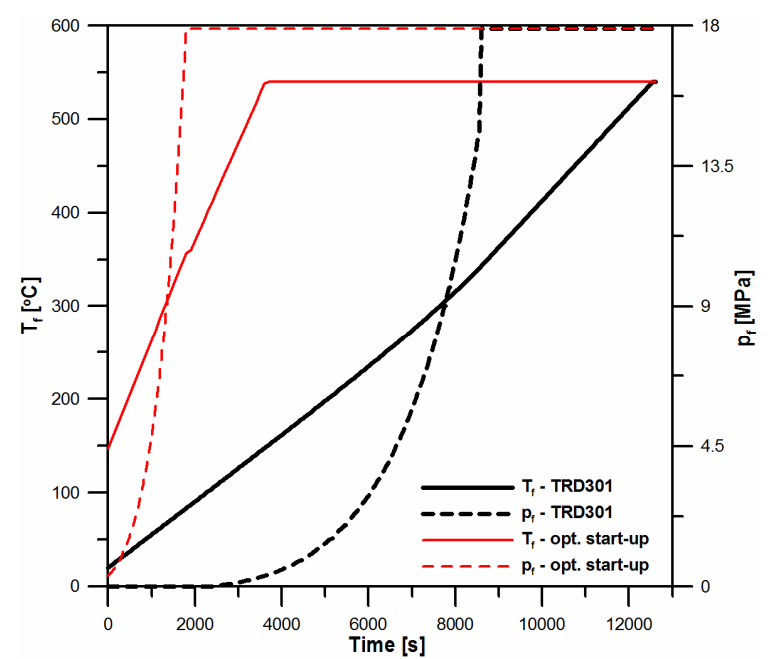

Fig. 9. Start-up temperature and pressure transients based on TRD301 regulations and optimization method.

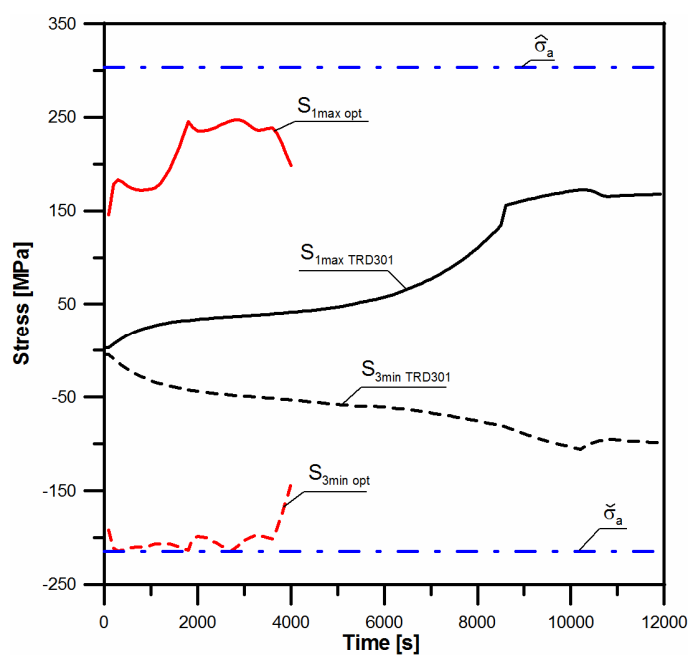

Fig. 10. Maximum first and minimum third principal stresses in the whole structure during start-up operation based on TRD301 regulations and optimization method.

Next, the cooling process optimization is carried out. The shut-down process time is minimized with the constraint $\hat{\sigma}_{a}=303 \mathrm{MPa}$. Vector of searched parameters $\mathbf{x}$ consists of $\left\{T_{f_{1}}, v_{T_{1}}, T_{f_{2}}, v_{T_{2}}\right\}$. For the lower and upper bounds of the searched-for parameters the values of $\left\{0^{\circ} \mathrm{C},-40 \mathrm{~K} / \mathrm{min}, \quad 0^{\circ} \mathrm{C},-40 \mathrm{~K} / \mathrm{min}\right\}$ and $\left\{600^{\circ} \mathrm{C}\right.$, $\left.0 \mathrm{~K} / \mathrm{min}, 600^{\circ} \mathrm{C}, 0 \mathrm{~K} / \mathrm{min}\right\}$ are selected. The starting vector is assumed as $\left\{400^{\circ} \mathrm{C},-4 \mathrm{~K} / \mathrm{min}, 300^{\circ} \mathrm{C},-4 \mathrm{~K} / \mathrm{min}\right\}$. Using the proposed optimization method the following parameters are found $\left\{472.2^{\circ} \mathrm{C},-5.91 \mathrm{~K} / \mathrm{min}, 324.2^{\circ} \mathrm{C}\right.$, $10.6 \mathrm{~K} / \mathrm{min}$ \}. Shut-down time is reduced from $12450 \mathrm{~s}$ to $3022 \mathrm{~s}$ as can be seen in Fig.11. The shut-down operation based on the proposed optimization method causes higher stresses than the cooling by TRD301, but all stresses are limited to the allowable values as can be seen in Fig. 12.

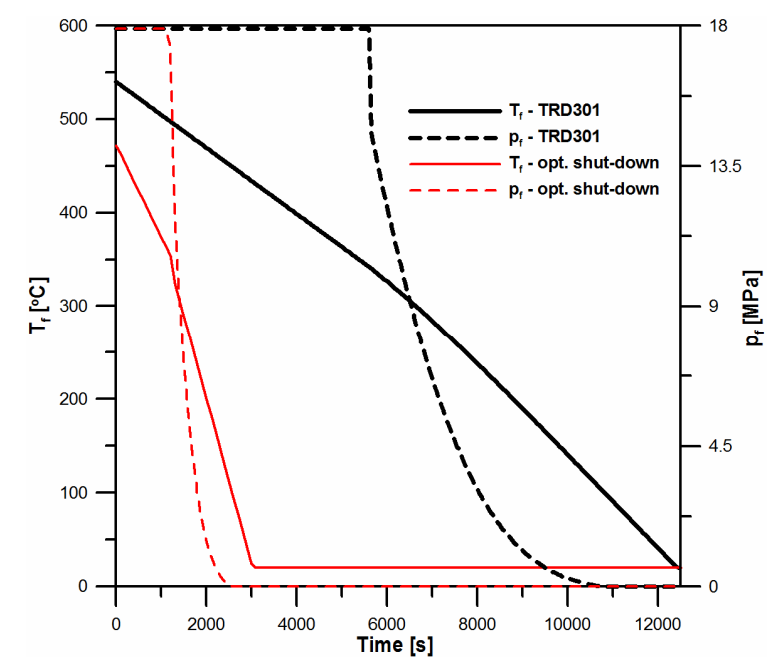

Fig. 11. Shut-down temperature and pressure transients based on TRD301 regulations and optimization method.

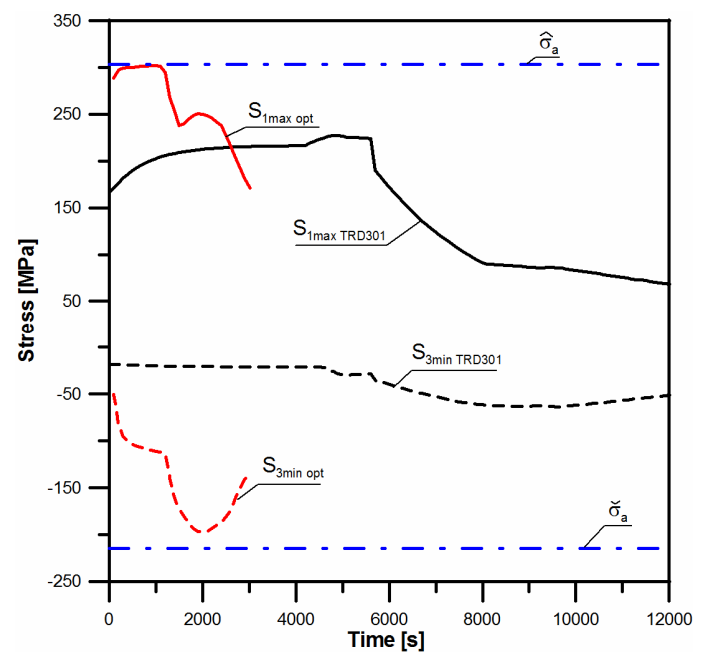

Fig. 12. Maximum first and minimum third principal stresses in the whole structure during shut-down operation based on TRD301 regulations and optimization method.

\section{Conclusions}

The work presents a two-stage methodology and a successful application of structural optimization of a Tpipe under transient thermal and mechanical loads. Genetic algorithms are applied in the first stage for the component shape modification. The optimization problem is defined to minimize the volume (mass) of the structure with stress constraints. The obtained geometry is about $10 \%$ lighter than the original one because the modified T-pipe wall thickness is smaller. This result could be considered as significant if bearing in mind that this structure has been used for many years and was probably already optimized by the designers. The maximum tensile and compressive stresses in the modified geometry are smaller than in the original $\mathrm{T}$ pipe during the whole cycle. Additionally, lower total stress values are recorded during heating and cooling processes. Therefore these transient processes can be accelerated and the shut-down and start-up losses can be reduced. In the second stage, the optimization of the 
modified T-pipe start-up and shut-down operations is conducted. The problem is defined to minimize the time of start-up and shut-down processes with stress constraints. The time of the start-up and shut-down processes is reduced from $12600 \mathrm{~s}$ to $3622 \mathrm{~s}$ and from $12450 \mathrm{~s}$ to $3022 \mathrm{~s}$ respectively. The total stresses, which are recorded during these operations, do not exceed the allowable values. Transient heating and cooling operations can be accelerated without decreasing the longevity of the T-pipe.

The proposed methods can be applied throughout the industry, wherever heating and cooling processes take place. The techniques used to find the optimum layout of the structure, optimum start-up and shut-down transients are fast and do not require a high financial outlay. The presented methods can be used for supercritical power units. The obtained optimization results translate directly into the power unit energy effectiveness, because the shut-down and start-up losses are reduced.

This research was financed by the National Science Centre, Poland, UMO-2015/19/B/ST8/00958

\section{References}

1. T.S. Kim, D.K. Lee, S.T. Ro, Applied Thermal Engineering 20, 11, 977-992 (2000)

2. P. Duda, R. Dwornicka, Structural and Multidisciplinary Optimization 40, 529-535 (2009)

3. P. Duda, D. Rząsa, International Journal of Energy Research 36 703-709 (2012)

4. K. Krüger, M. Rode, R. Franke, Energy 29, 12-15, 2239-2251 (2004)

5. TRD 301, Technische Regeln für Dampfkessel, Carl Heymans Verlag, Köln und Beuth-Verlag, Berlin (2001)

6. R.J. Yang, S.C. Poe, Structural Optimization 5 233239 (1993)

7. R.V. Tappeta, S. Nagendra, J.E. Renaud, Structural Optimization 18 134-145 (1999)

8. T. Wen et al., International Journal of Heat and Mass Transfer 50 2590-2604 (2007)

9. M. Yoon, S.-H. Ha, S. Cho, International Jour-nal of Heat and Mass Transfer 62 272-285 (2013)

10. G.N. Xie, Q. Wang, Applied Thermal Engineering 59 425-434 (2013)

11. C.L. Xiao, H.X. Huang, Applied Thermal Engineering 64 462-470 (2014)

12. G. Nowak, A. Rusin, Energy Conversion and Management 74 417-425 (2013)

13. K. Schittkowski, Annals of Operations Research 5 Issue 1-4, 485-500 (1986)

14. A. Osyczka et al., Evolutionary optimization system (EOS) for design automation. In: Burczynski T, Osyczka A (eds) Evolutionary methods in mechanics. Kluwer, Dordrecht (2004)

15. M. Mrzyglod, A. Osyczka, Parallel Computing for Design Optimization with Computationally
Expensive Functions us-ing Evolutionary Algorithms (in B.H.V. Topping, (Editor), Proceedings of the Eighth International Conference on the Application of Artificial Intelligence to Civil, Structural and Environmental Engineering, CivilComp Press, Stirlingshire, UK, Paper 26, 2005)

16. F. Richter, Physikalische Eigenschaften von Stählen und ihre Temperaturabhängigkeit. Mannesmann Forschungberichte 930, Düsseldorf (1983)

17. ANSYS Theory Guide, Release 14, (2013)

18. J. Taler, P. Duda, Solving direct and inverse heat conduction problems, Springer-Verlag, Berlin, (2006) 